\title{
Novel approach to determine age and gender from wrist $x$-ray images
}

\author{
Santosh K C $\mathbf{C}^{\mathrm{a}}$, Pradeep $\mathbf{N}^{\mathrm{b}}$ \\ a Assistant Professor, CS\&E department, B.I.E.T., Davangere, Karnataka, India \\ ${ }^{\mathrm{b}}$ Associate Professor, CS\&E department, B.I.E.T., Davangere, Karnataka, India
}

Article History: Received: 10 November 2020; Revised 12 January 2021 Accepted: 27 January 2021; Published online: 5 April 2021

\begin{abstract}
Human gender and age prediction in field of forensic department is a very important and crucial stage in means of criminal and judicial law. Human identification is essential when it required for recognising a body in case of mass disaster and natural disaster like earth quake, floods, tsunamis, hurricanes and other geological process that causes huge damage for mankind and loss of life. Human bones during the growth stages undergo few substantial changes of size and shapes. In diagnosing growth of bones, $\mathrm{x}$ ray images are frequently used. Hand $\mathrm{x}$ ray images in particular has been chosen as a part of $\mathrm{x}$ ray imaging, since hand has more unique features and more number of parts. Manual technique of identification is also attainable, but this process can be adopted when medical practitioners, assistants and basic tools are available. Manual method can be carried out based on the availability of bone like skull, long bones, short bones, hand, pelvis bone etc. It requires ample time to process the accurate outcome of the available samples. Hence hand operated technique is not feasible for identification. A machine driven automated system for gender and age identification is essential to overcome the flaws occurred in manual technique. This would facilitate better outcome in lesser time, without intervention of labour and also enables quantitative and accurate assessments. In the proposed system, we have identified most important features from wrist bone which contributes in age and gender identification. Main aim of our study is to identify gender and estimation of age of Middle East population of Karnataka state in India by analysing digital images of wrist bone. Random forest classification algorithm is used to deploy this system by considering 76 male samples and 50 female samples in total 126 wrist radiographs of age group between 06 to 78 years old. Random forest classifier belongs to decision tree family, each decision tree when executed may tends to overfit that training data, but random forest avoids this over fitting problems and it will try to capture maximum amount of pattern. Since multiple decision trees are implemented in RFC, this makes it a power full classification algorithm that will predict results with higher accuracy most of the time. Accuracy of $97 \%$ is achieved in the present work for age and gender prediction.
\end{abstract}

Keywords: forensic, wrist bone, gender identification, age estimation.

\section{Introduction}

Human bones are subject to undergo changes in shape and size throughout they exist in the lifespan. Gender identification with age estimation plays an important role and challenging task in forensic field. Bone age analyses are frequently done in youngster patients to predict their deficiencies in growth and to detect and manage endocrine disorders. Over decades, evaluation of bone growth was based on visual method for hand and wrist bone is commonly done using two standard method: i) Greulich and Pyle atlas method and ii) Tanner Whitehouse method. These two methods take left hand digital images as input for prediction which is totally dependent on professional expert who checks input radiograph with standardised atlases to identify any differences if exists, later they assigns evaluated score to radiograph. On an average a trained expert radiologist may take around 15 minutes for prediction of gender and age of an input image. These manual prediction techniques consume more time and they are subject to produce differences in prediction based on the observers. Literally, the accuracy and robustness of this technique is low because of the bias in intra and inter observers, since the laboratory experience plays vital role for reporting the correct estimation.

Predictions based on atlas methods need great labour effort to judge and compare radiograph for references from atlas images [1]. This process is also subjective that different evaluators judge in different approach based on their training and expertise level in this field. With the advancement in digital technology and digital imaging, many attempts was done to deploy a standard image processing method that will automatically explore key features from bone ossification that provides more efficient and effective approach in assessment of bone maturity. To the best of our knowledge, there are no standard parameters and tools reported yet that will identify gender with age by the input of hand wrist radiographs. The changes occurs commonly in human bone that particularly greater during growth period. Hand bone is closely joined to the lower arm through radius and ulna bones. It is made up of metacarpal and phalange bones, 8 carpal bones all together 27 small bones. These arrangements of wrist hand bones or anatomy of bones are depicted in figure 1. 


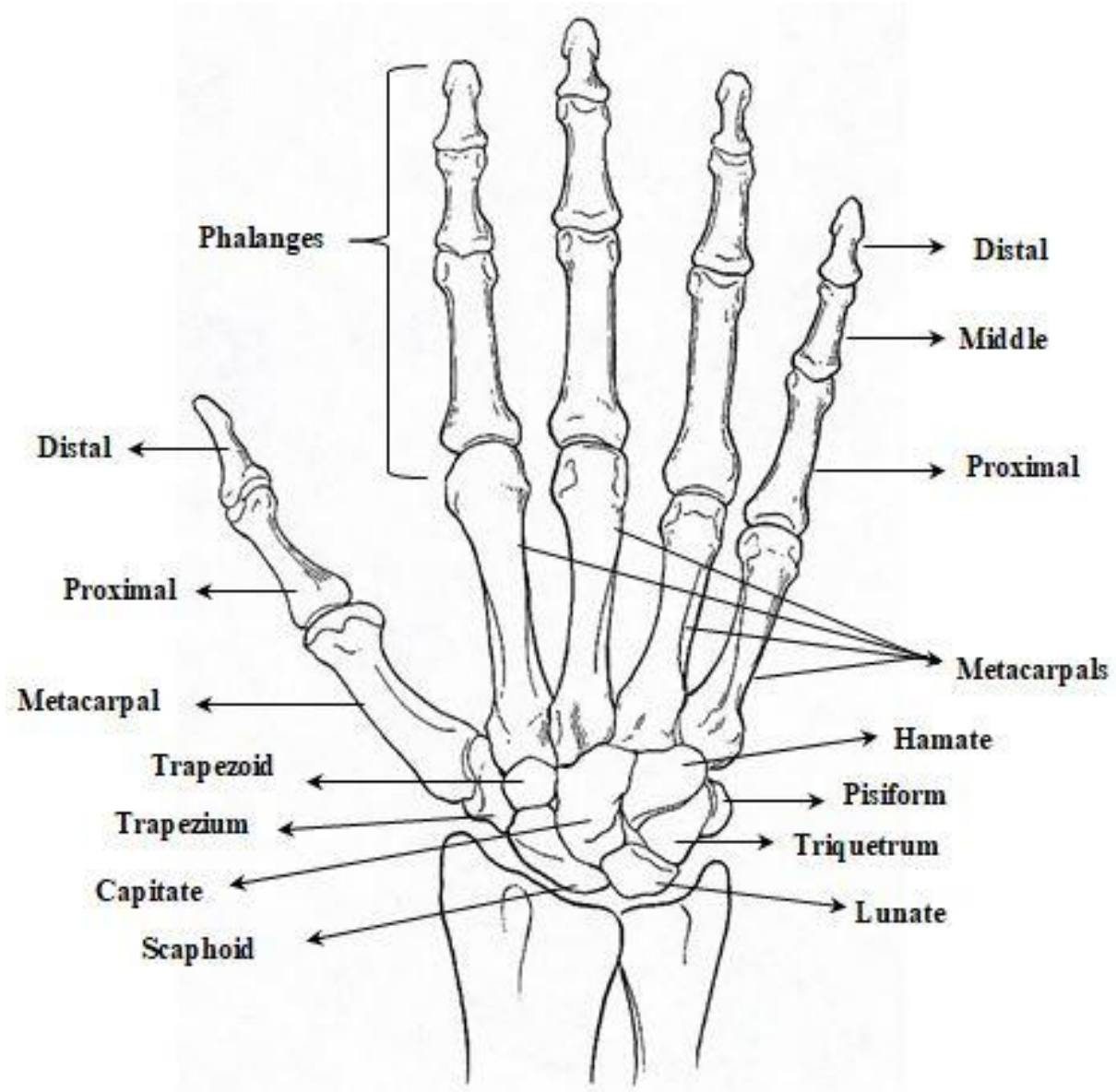

Figure 1: Anterior view of hand wrist bone

The appearance of eight carpal wrist bones in human hand in chronological order with respect to age along with gender is depicted in table 1 below. Hamate and Capitate are the two carpal bones of the wrist that appears in new born baby, Triquetrum bone can be visible first in a girl infant baby. Appearances of carpal bones are delayed in boys compared to girls.

Table 1: Appearance of carpal bones chronologically.

\begin{tabular}{|c|c|c|c|}
\hline $\begin{array}{c}\text { Bone } \\
\text { appeared }\end{array}$ & $\begin{array}{c}\text { Carpal } \\
\text { bone }\end{array}$ & Female & Male \\
\hline First & Capitate & 2.0 months & 4.0 months \\
\hline Second & Hamate & 2.0 months & 4.0 months \\
\hline Third & Triquetrum & 2 yrs & 3 yrs \\
\hline Fourth & Lunate & 3 yrs & 4 yrs \\
\hline Fifth & Trapezium & 3 yrs & 4 yrs \\
\hline Sixth & Trapezoid & 4 yrs & 6 yrs \\
\hline Seventh & Scaphoid & 4 yrs & 6 yrs \\
\hline Eighth & Pisiform & 9 yrs & 12 yrs \\
\hline
\end{tabular}

Lunate and trapezium bones are appeared around the age of 3 - 4 years of kids. Sixth and seventh carpal bones trapezoid and scaphoid appears at the age of 4- 6 years. At last pisiform bone appears around 9 years of age in girl $\mathrm{s}$ and 12 years in boys. 
In past few years, many methods for identification of gender and age were proposed, most of these approaches based on certain feature extraction from the image and classification technique. We implement the automatic gender with age identification system using random forest algorithm for high accuracy in prediction [2].
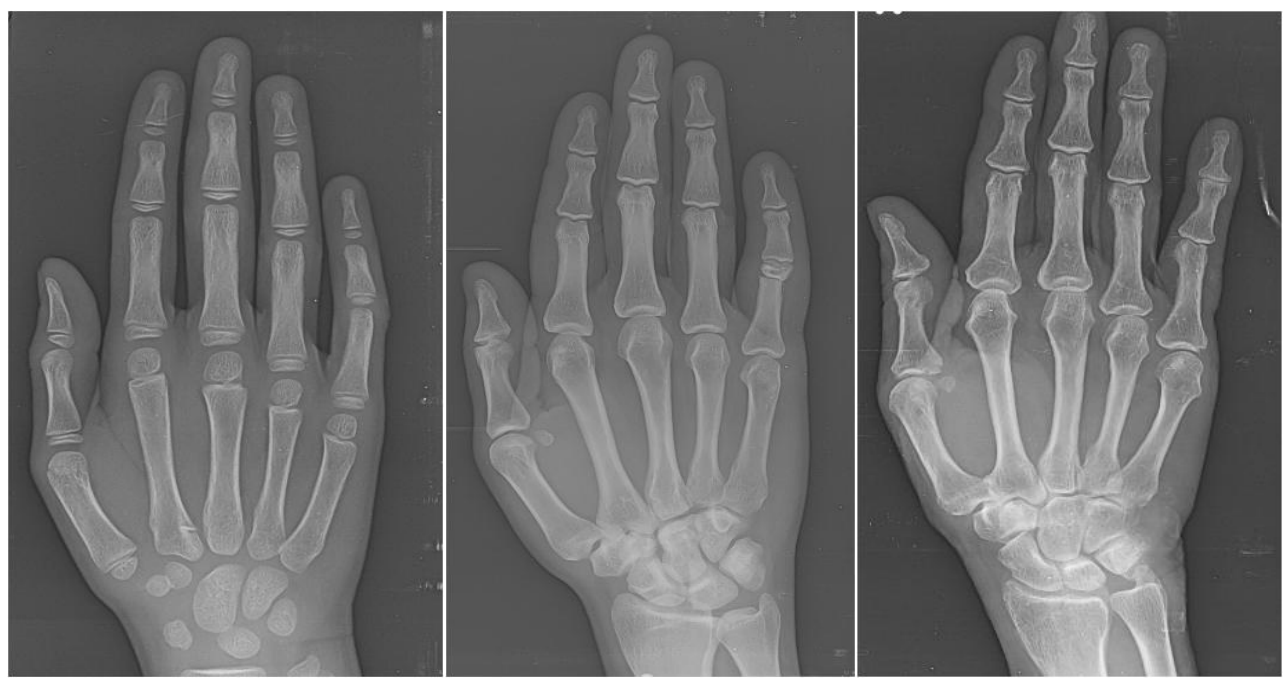

Figure 2: Hand wrist images of different age and gender

Hand wrist images of various age and gender are depicted in figure 2. Size and shape of carpal bones are different from one hand image to other. During early childhood, the carpal bone appearance and growth differs from developmental stage. Another important change in shape for age factor is ossification, especially cartilage of epiphyses part turns to hard bones. Bone age evaluation is usually done based on a digital image of left hand part, because it is a trustable indicator of gender and age prediction. Generally right hand is dominant in physical activity of human and it is subject to some accidental injuries. Age variation of human can be identified based on growth plates in the palms from an $\mathrm{x}$ ray image of hand. Initially growth plates will be thinner as the child grows to adulthood, the plates will disappear. Hand wrist $\mathrm{x}$ ray images are commonly used for estimation of bone age since hand can be easily exposed for minimal $\mathrm{x}$-ray radiation with any position and direction, it also include many bones in single view [3].

For ease of bone age analysis, we divided development of bone into five important stages and specific regions for identification which are best indicator of every group-

- $\quad$ Early childhood: appearance of carpal and radial epiphyses.

- Kids: Visibility of epiphyses in long bone.

- $\quad$ Pre puberty stage: Phalangeal epiphyses size.

- $\quad$ Late puberty stage: Fusion of epiphyses.

- $\quad$ Post puberty stage: fusion of epiphyseal of ulna and radius. 


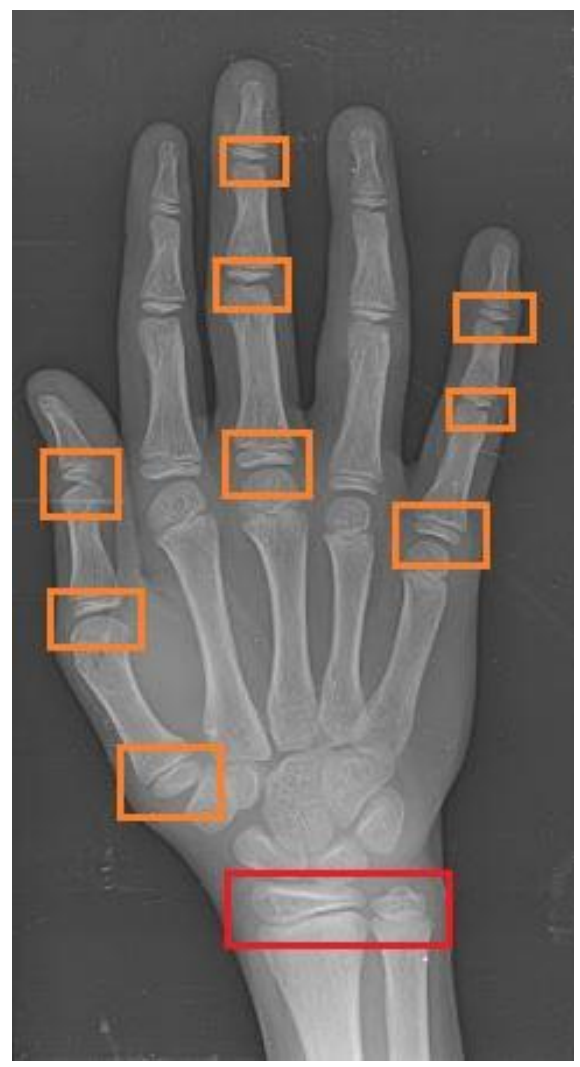

Figure 3: ROI for TW method predictor.

Tanner and Whitehouse technique is used in manually prediction of age and gender. RoI identified in TW evaluation technique is depicted in figure 3. Some regions are marked in orange colour boxes and red coloured box. The bone growth plates are indicated in the phalanges in orange colour boxes and the carpal bones section which are attached to radius and ulna are indicated in red box. Instead of looking entire hand $\mathrm{x}$ ray image, TW method looks only particular areas in the $\mathrm{x}$ ray to avoid the complexity and assessment subjectivity. The main areas in the hand image as marked in figure 3 are metaphysis and epiphysis from phalanges and carpal bones. In particular, three fingers from phalanges were analysed i.e. middle finger, pinkie finger and thumb finger.

\section{Literature Review}

Identifying the gender of human with age is a challenging and time consuming process if it was done manually. Hence a fully automated solution for identification was developed. In this section the basic idea, motivation and the methods adopted by other researchers have been summarized. Their contribution towards forensic medical field through Computer Vision, Artificial Intelligence, Machine Learning and Deep Learning was briefed accordingly:

Mohd Asyraf Zulkifley et. al. [4] in 2020 developed an automated system for bone age prediction with image regression technique based on wrist radiographs. Their model based on regression model that uses separable residual convolution method. It requires an image of 199 x 199 pixels, which is mapped for predictable bone age from various modules in XCeption network. Before providing image for prediction, it has to be registered or preprocessed to a normalized and standardized form with separable CNN. Their experiment outcome reflected that lowest average absolute error is 8.21 months and average squared error rate is 122 months. Error which is less than a year can be accepted for prediction of age, this can be implemented as alternate tool for second advice for prediction.

Christian Booz et. al. [5] in 2020 evaluated a method based on AI for assessment of bone age. Their team analysed efficiency and accuracy of an AI based software dedicated for assessment of bone age by comparing GP technique. They have compared a sample of 524 radiographs in their study. Bone age estimation was carried by four experts by applying GP technique for AI software. Three blinded experts results were compared with other reading, the differences were observed that in terms of accuracy and time efficiency, software based method found to be applicable in various field. 
Roberto Scendon et. Al. [6] in 2020 analyzed carpal bones based on MR images estimation of human age. Their purpose of present study is to build a method for estimation of age from the average obtained from ossification of nucleus and the growth surface of every carpal. They analysed 58 magnetic resonance imagingMRI radiographs of age grouped 11 years to 19 years without any pre-existing disease. Parameters of ossification of nucleus and growth surface were measured using software ImageJ. Their outcome depicts that best and accurate results gained from five features predictors like ossification of nucleus and growth surface of capitate, scaphoid, trapezium, trapezoid and pisiform.

Jang Hyung Lee et. al. in [7] 2020 proposed a method for estimation of bone age based on digital images of wrist by adopting deep learning techniques. They implemented a system with a set of deep learning architecture with around 3000 digital images were used for marking the feature point on wrist image. To retain region of interest, these points were considered as base points for estimation of age. The factors which are not relevant to age estimation can be preserved by selecting suitable part from the wrist images in order to reduce complexity. Since the background region in a digital images are not useful in determination of age and hence that may be removed without effecting to system performance.
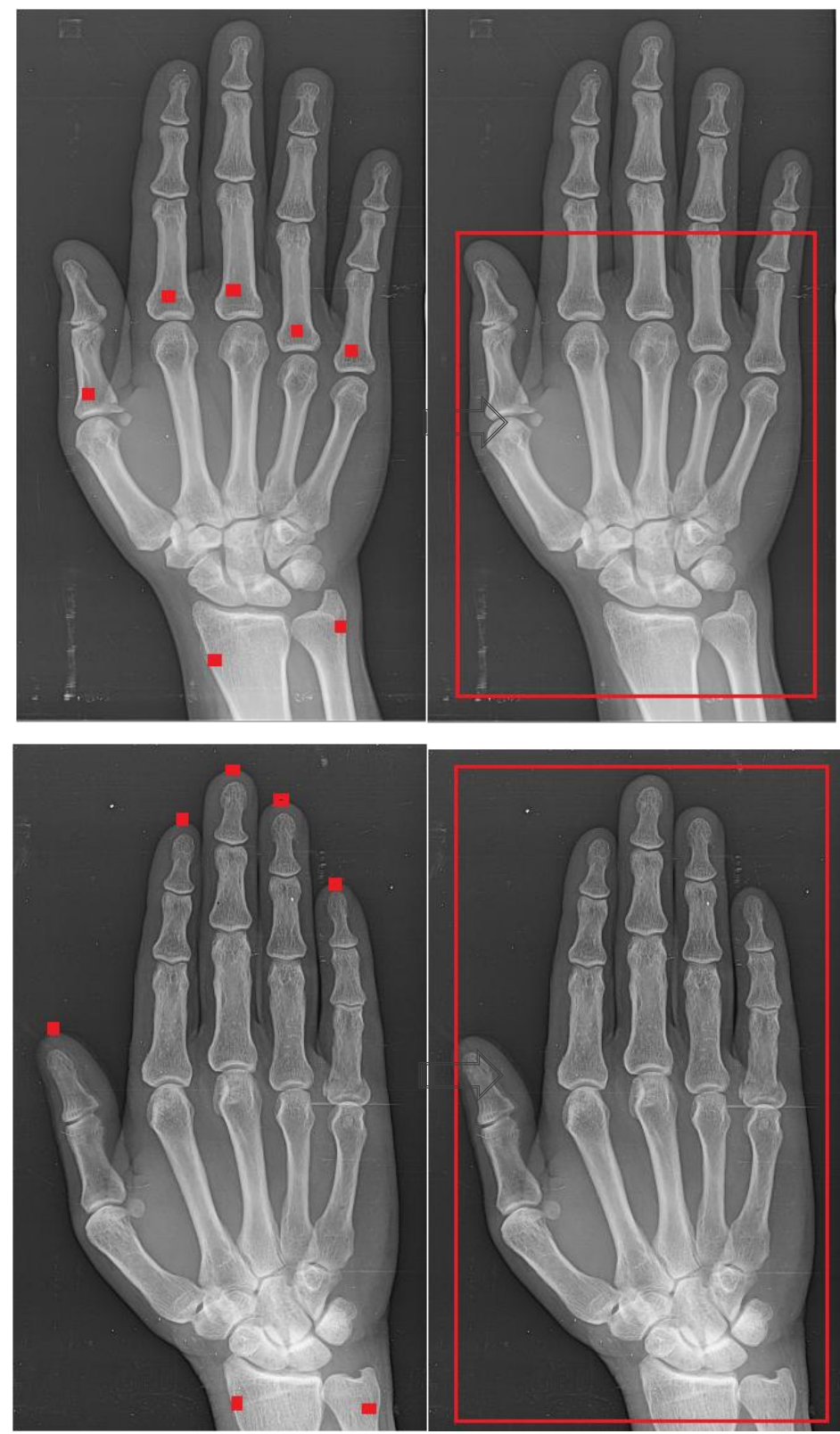

Figure 4: Features point indication on left images and ROI at right images.

Closely related feature pattern may occur in hand images which are not required, can be removed from estimation of age process. This method is depicted in figure 4. Rectangle shaped red coloured box defines the largest area to be in the hand image which can be used as region of interest in identification process. Fingers 
represent wide range of variance than palm. A group of points which are marked in phalanges ulna and radius can be used as an input criterion in order to minimise finger based variations.

Ana Luiza Dallora et. al. [8] in 2019 reviewed several machine learning methods related to identification based on wrist bone. They presented a systematic review as a proof and evidence for the estimation, also to provide as a material to fill gap for the age and gender assessment related issues. Their review begins with a protocol on searches based on three database: Scopus, Web of Sciences and Pubmed relatively to identify interrelated connections with estimation of bone age based on ML methods. Few studies are selected to check the quality of assessments and to analyse biased on lower impact studies. Authors analysed and compared 25 studies for their final estimation, out of theses many of them presented automated system for gender and age estimation techniques based on wrist digital images.

Sehyo Yune et. al. [9] in 2019 discovered a method for gender determination based on children radiographs of wrist. They trained images and tested them adopting deep convolution neural network-CNN methods to get exposer from deep learning algorithm that can play important role in identifying the differences from medical images that were not able to identify by professionals in radiology. They used samples of 8000 wrist digital images of age 5 - 18 years, they were selected $5 \%$ of images randomly for validation purpose, and $80 \%$ of images were used for training purpose and remaining $15 \%$ used for testing. Those trained images were tagged individually with their gender along with age. The average bone age of datasets predicted by the model is 11 year 07 month. They also noticed that in majority of girls samples used for prediction was focused on carpal bones and boys samples used for age identification were based on metacarpal bones in maximum cases.

Akanksha Sharma and Prabhjeet Kaur [10] in 2019 developed a method for estimation of age using hand wrist radiographs. They implemented this system using $C N N$ algorithm designed Matlab tool of version 2016a. Bone age estimation was based on framework of graphic user interface and $P-C N N$ method was used for classification purpose. Practical Swarm optimization-PSO is used in training the model as well as to minimize many classification methods. They have used 45 digital labelled images of wrist for their model to extract the dominant features that will help the system to estimate age accurately. Using filtration and optimization technique, features vector were analysed and selected from wrist bone radiographs. Overall results were evaluated on the basis from true negative, true positive, false negative and false positive by determining specific accuracy point.

Table 2: Summary of Literature survey.

\begin{tabular}{|c|c|c|c|c|}
\hline Sl. No. & Authors & Year & Research findings & Remarks \\
\hline 01 & $\begin{array}{l}\text { Mohd Asyraf Zulkifley, Siti Raihanah } \\
\text { Abdani and Nuraisyah Hani Zulkifley [3] }\end{array}$ & 2020 & $\begin{array}{l}\text { Lowest average absolute } \\
\text { error is } 8.21 \text { months and } \\
\text { average squared error rate } \\
\text { is } 122 \text { months. }\end{array}$ & $\begin{array}{l}\text { Can be implemented as } \\
\text { alternate tool for predicition. }\end{array}$ \\
\hline 02 & $\begin{array}{l}\text { Christian Booz, Ibrahim Yel1, Julian L. } \\
\text { Wichmann [4] }\end{array}$ & 2020 & $\begin{array}{l}\text { Reviewed several method } \\
\text { based on AI for } \\
\text { assessment of bone age. }\end{array}$ & $\begin{array}{l}\text { Software based method found } \\
\text { to be applicable in various } \\
\text { field. }\end{array}$ \\
\hline 03 & $\begin{array}{l}\text { Roberto Scendon } \\
\text { Mariano Cingolani, Andrea Giovagnoni, } \\
\text { Marco Fogante, Piergiorgio Fedeli, Yu. I. } \\
\text { Pigolkin, Luigi Ferrante, Roberto } \\
\text { Cameriere [5] }\end{array}$ & 2020 & $\begin{array}{l}\text { Carpal bones evaluation } \\
\text { based on MR images. }\end{array}$ & $\begin{array}{l}\text { Ossification of nucleus and } \\
\text { growth surface of capitate, } \\
\text { scaphoid, trapezium, trapezoid } \\
\text { and pisiform are best } \\
\text { parameters for prediction. }\end{array}$ \\
\hline 04 & $\begin{array}{l}\text { Jang Hyung Lee, Young Jae Kim, Kwang } \\
\text { Gi Kim. }\end{array}$ & 2020 & $\begin{array}{l}\text { A group of points in } \\
\text { fingers represent wide } \\
\text { range of variance than } \\
\text { palm. }\end{array}$ & $\begin{array}{l}\text { Used to mark the feature point } \\
\text { on wrist image. }\end{array}$ \\
\hline 05 & $\begin{array}{l}\text { Ana Luiza Dallora, Peter Anderberg, Ola } \\
\text { Kvist. }\end{array}$ & 2019 & $\begin{array}{l}\text { Systematic review as a } \\
\text { proof and evidence for the } \\
\text { estimation of age based } \\
\text { on wrist. }\end{array}$ & $\begin{array}{l}\text { To fill the gap in age and } \\
\text { gender assessment related } \\
\text { issues. }\end{array}$ \\
\hline 06 & $\begin{array}{l}\text { Sehyo Yune, Hyunkwang Lee, } \\
\text { Myeongchan Kim, Michael Gee. }\end{array}$ & 2019 & $\begin{array}{l}\text { The average bone age was } \\
11 \text { year } 07 \text { months with } \\
91.1 \% \text { accuracy. }\end{array}$ & $\begin{array}{l}\text { Training and testing images by } \\
\text { adopting deep convolution } \\
\text { neural network-CNN methods }\end{array}$ \\
\hline 07 & Akanksha Sharma and Prabhjeet Kaur & 2019 & $\begin{array}{l}\text { Accuracy in bone age } \\
\text { estimation is } 96 \%\end{array}$ & $\begin{array}{l}\text { Designed Matlab tool of } \\
\text { version 2016a }\end{array}$ \\
\hline
\end{tabular}

\section{Feature Identification}

Wrist hand bones contribution in age and gender identification plays an important role since it has more unique features than any other part in human. When a digital image of wrist part is available, more importantly the ossifications of carpal bones are observed and the growth plates in between phalanges is also important consideration in identification. Adult human body contain 206 bones, whereas hands and feet contribute more than $50 \%$ of bones. Since a hand bone has 27 small bones like 08 carpal bone, 5 distal bones, 5 proximal bones, 4 middle phalanges and 5 metacarpal bones and hence identification of gender with age estimation from a hand wrist digital image is more challenging task. The process of growth can be evaluated from these bones that 
appearance from calcification point to epiphyseal growth plates closure occurrence throughout their growth period, hence wrist bones provide a valuable means of skeleton maturity.

Some of the salient features of wrist that contributes in identification of gender and age are discussed in this section. The features are identified and feature values extracted by the system and stores in the database for further prediction. Important features from hand wrist are as follows:

- Carpal bone: Appearance of carpal bone from an infant to adulthood is a good indicator for age estimation with a difference of 4-5 months. Figure 5 and figure 6 depicts the age of input image based on carpal bone of wrist.
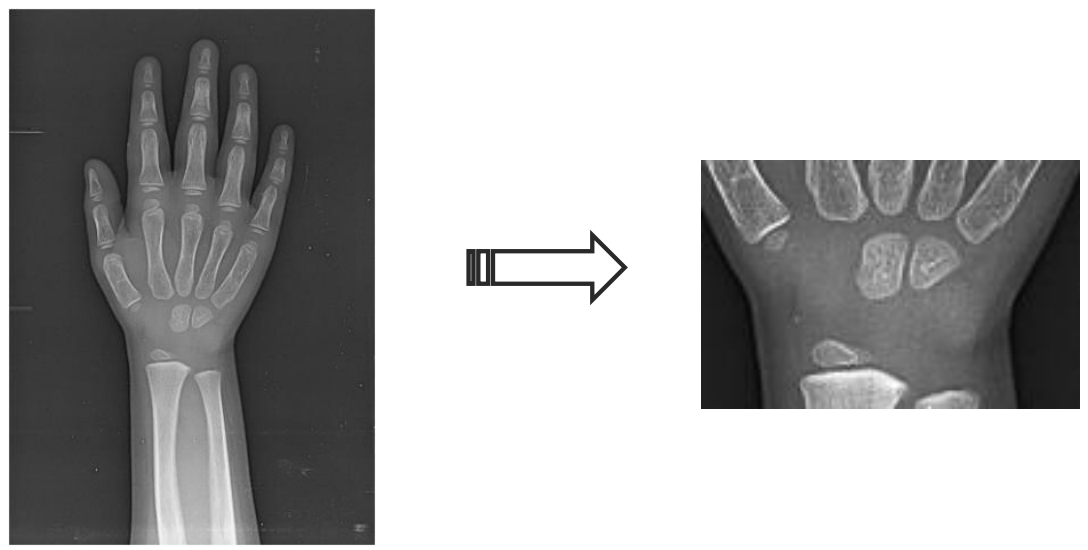

Figure 5: Appearance of carpal bones at age 4 years.
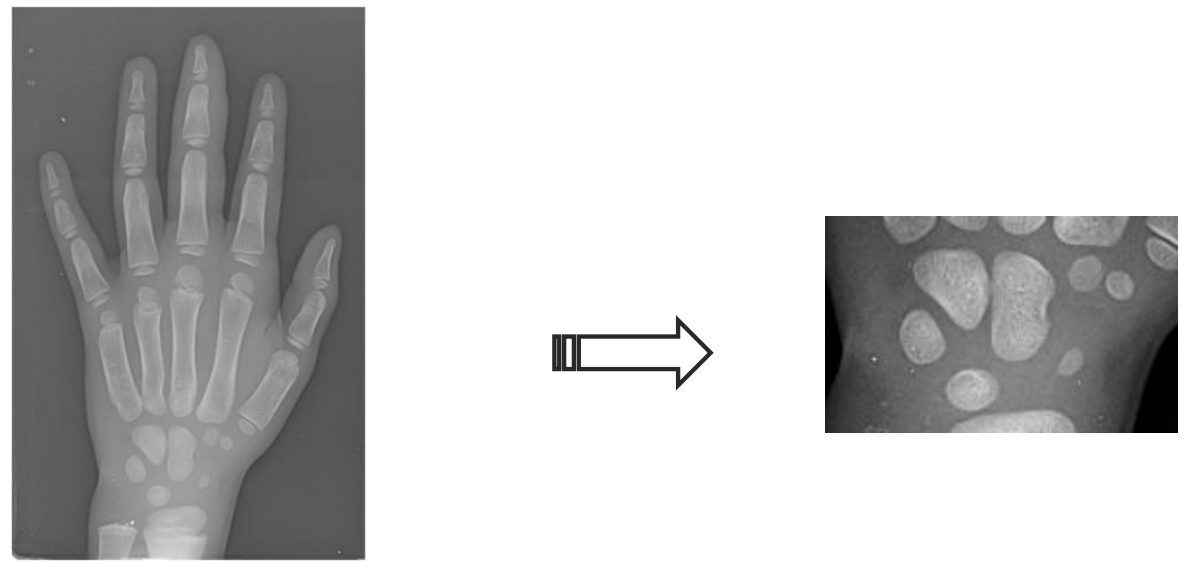

Figure 6: Appearance of carpal bones at age 9 years.

- Epiphyseal plate fusion: Epiphyseal plates also known growth plates is a good indicator of maturity. Growth plate at the proximal end of metacarpal stops growing at certain age, in between 14 to 16 years old. Figure $7(a)$ and 7 (b) depicts fusion of growth plates at different stages. 

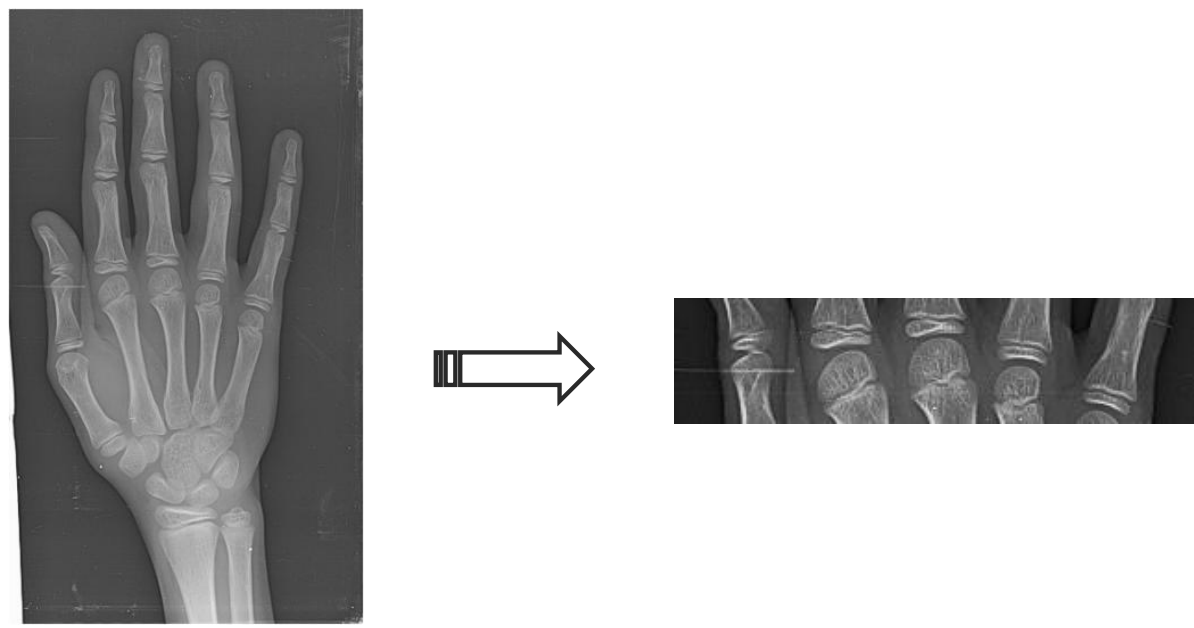

7 (a)
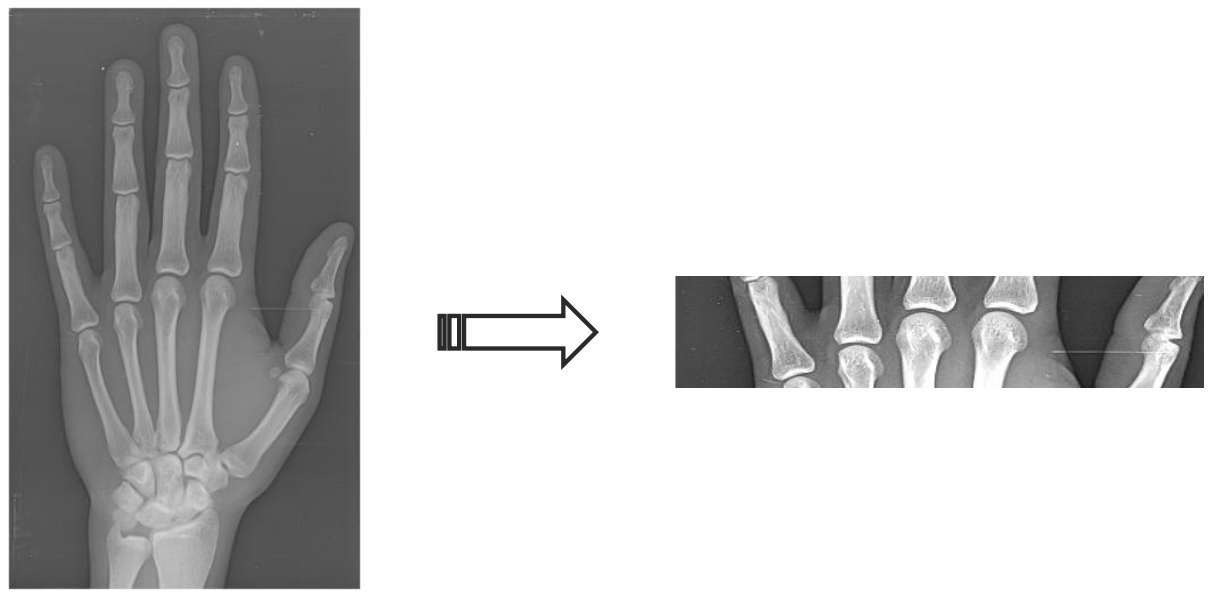

7 (b)

Figure 7: Epiphyseal plate fusion (a) at age 13 years (b) at age 27 years

- Sesamoid bone: Apart from knee, sesamoid bone is present at the bottom of thumb finger. It's a small rounded ball shape bone used to reduce the stress on tendon. Appearance of sesamoid bone in human starts at the age of 15 years. Figure 8 shows the presence of sesamoid bone which is a important feature for age detection.
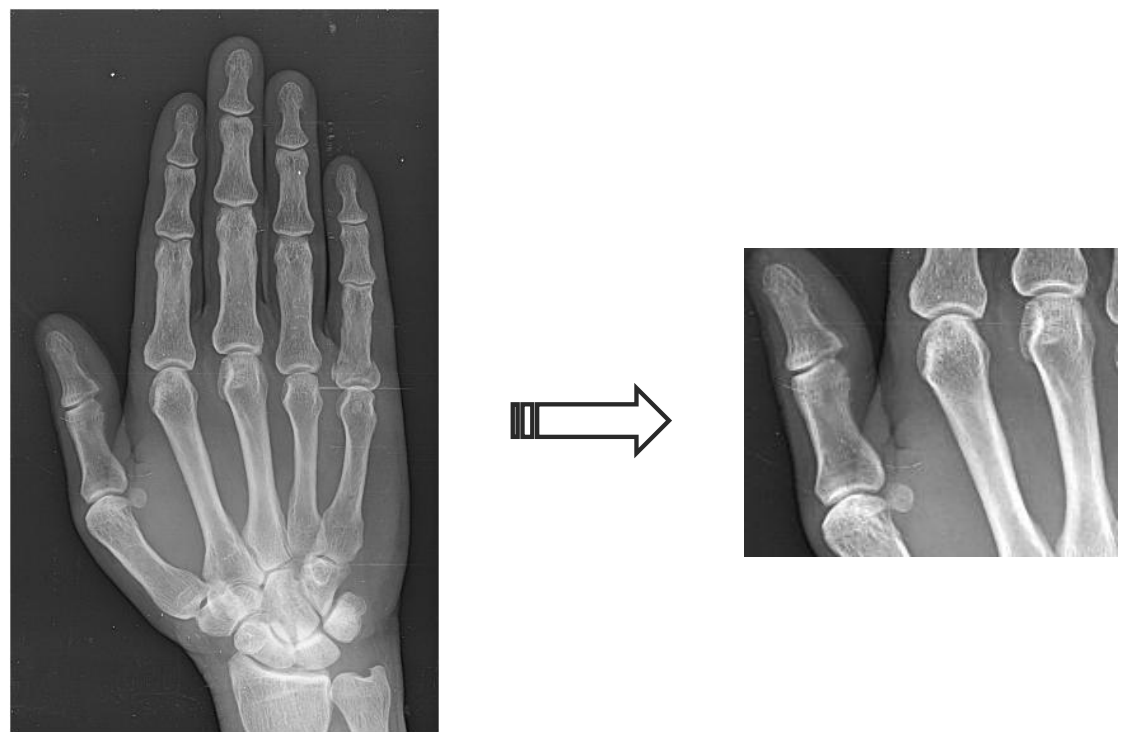

Figure 8: Appearance of sesamoid bone at age 21 years 


\section{Materials And Methods}

The current research study was based on the digital images of wrist bone. Digital x-ray images of wrist bone (datasets) were not publically available, hence we collected datasets from Department of Radiology, Jagadguru Jayadeva Murugarajendra Medical College, Davangere, Karnataka. Memorandum of Understanding (MoU) for academic cooperation has been established on 29-09-2018 between the research scholar and Jagadguru Jayadeva Murugarajendra Medical College, Davangere. A total 465 digital images of different parts of human were obtained, out of 465 clinical records 126 healthy images of wrist bones were selected and analysed, in which 106 samples used for training sets and 20 images used for testing phase.

The age distributions of available 126 datasets were divided into 13 groups with respect to gender of 5 years of range per group except last group, which is used for age group above 60 years. Figure 9 depicts distribution of datasets based on age of interval 5 years, segregating male and female count per group. In total there are 76 male and 50 female radiographs, which were collected for experimental analysis.

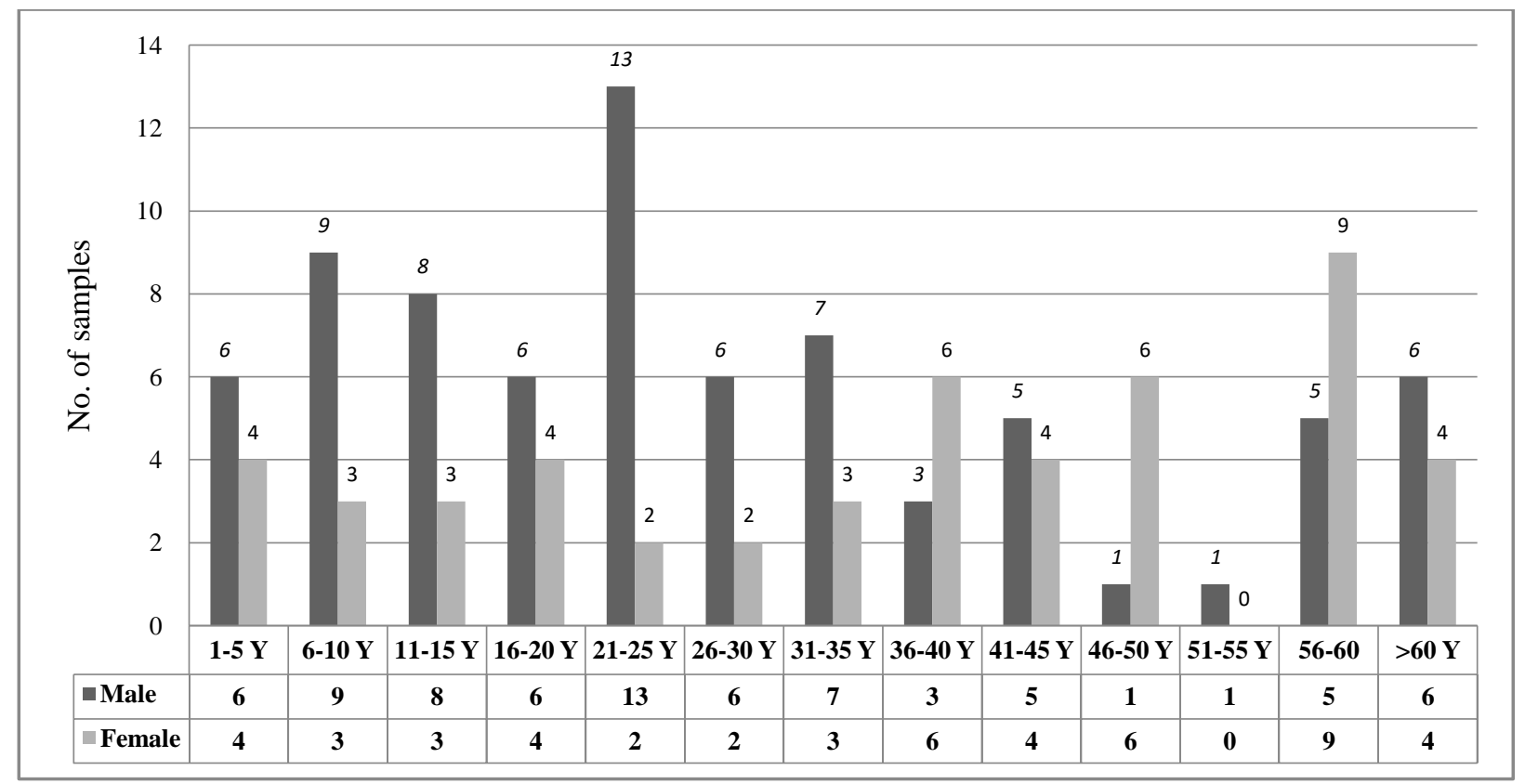

Figure 9: Age dataset distribution against gender.

In present research work, radiographs from patients with age of 02-77 years were included in the dataset. Manual process of age and gender identification requires considerably more time and background knowledge about anatomy. Hence we have developed a model that will identify gender of a person with estimation of age. Identification of gender and age estimation can be investigated using many imaging services available i.e. digital $\mathrm{x}$ ray radiographs, MRI radiographs or CT scanned images. Compared to CT scan images and MRI based imaging $\mathrm{X}$-ray radiographs provides a cost effective, painless and its process is simpler and fast. Hence we have collected digital x-ray images of wrist for experimental process. 

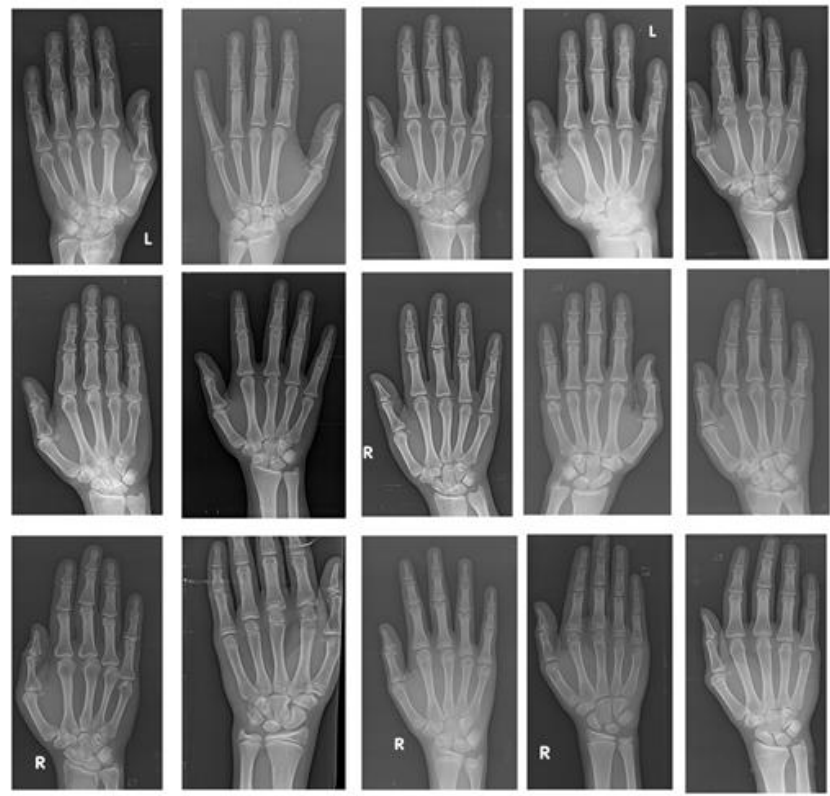

Figure 10: Input images collected from JJM medical college, Davangere.

Hand wrist bone (datasets) were not available publically as per our search, so we collected datasets from Department of Radiology, Jagadguru Jayadeva Murugarajendra Medical College, Davangere, Karnataka with legal consent letters. Few snapshots of data collected were depicted in figure 10.

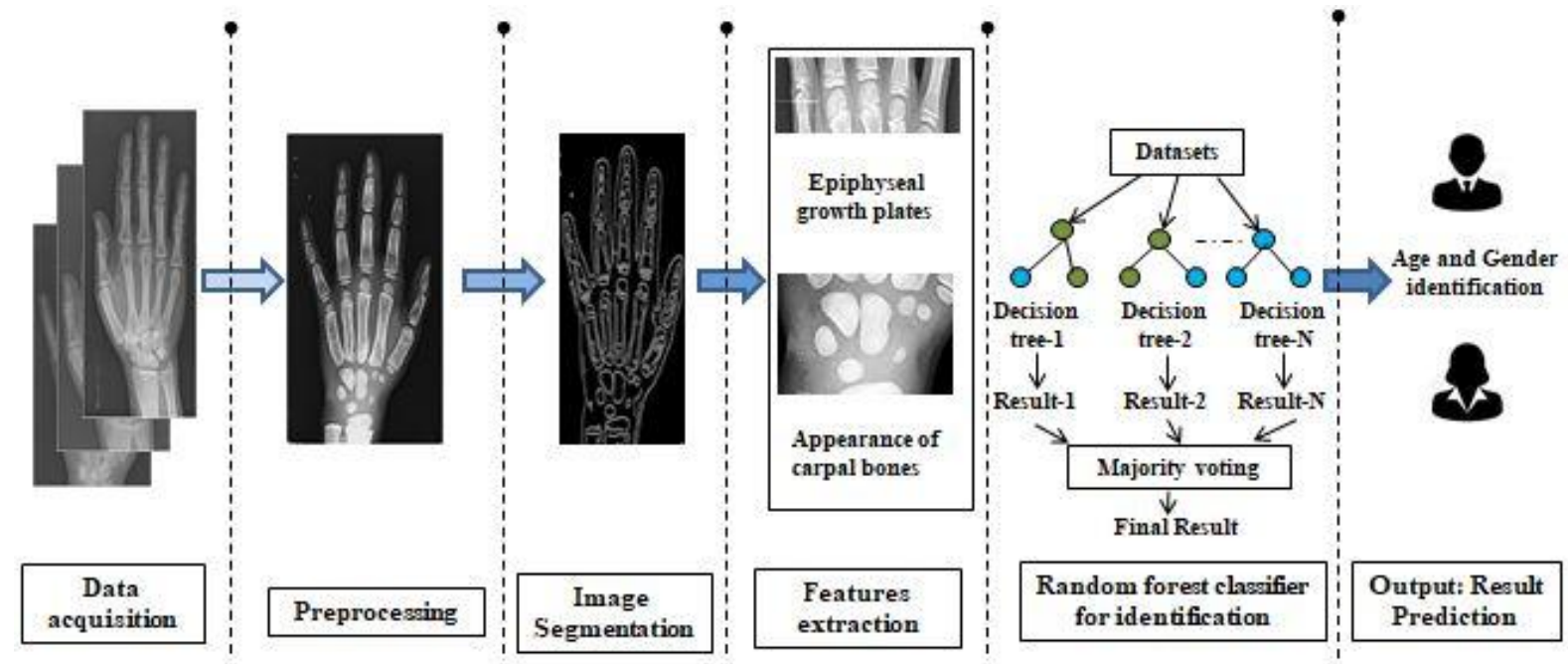

Figure 11: Age and gender identification system using wrist image.

Methodology of the proposed Gender and Age estimation system based on digital wrist x-rays is depicted in figure 11. The fundamental block are: Data Acquisition, Pre-processing, Image Segmentation, Feature Extraction and Random Forest for age and gender identification. The collected samples are distinguished as test case samples and training samples, for test cases we have used $20 \%$ of the samples and $80 \%$ of the remaining samples are used for training the model. A normal healthy hand wrist bone image is given as the input for the system, that image is pre-processed first by removing noise and unwanted labels in the image. The result of preprocessed image is an enhanced form of input image for a better accuracy of the prediction. Identifying bright pixels on the boundary of the image is the method of edge detection in medical images, by this few important features and characters were marked and analysed. For segmentation of the image, we have applied canny edge segmentation technique, and the result of this is depicted in the methodology diagram. Segmentation of a digital image is a method of separation of image from its background outliers. After the segmentation steps followed the system, the digital image may be subject to blurriness. This can be removed by varying brightness and contrast of gray scale, this process is known as smoothing technique of digital images. We have implemented the system by using one of the popular decision tree algorithm, known as Random Forest classifier. Particularly we stick on to random forest classifier, since its belong to decision tree family, and each decision tree when executed may tends 
to overfit that training data, but random forest avoids this over fitting problems and it will try to capture maximum amount of pattern. Since multiple decision trees are implemented in RFC, this makes it a power full classification algorithm that will predict results with higher accuracy most of the time.

\section{Experimental Results And Discussions}

We have proposed a fully automated system for age and gender identification, with the deployed system we can automatically able to predict age and gender of an input image. Our proposed Age and Gender identification system takes input of digital X-ray images of wrist bone. Digital X-ray images of hand wrist bones were collected from JJM medical college, Davangere, Karnataka. After an input is selected for prediction, first step is to perform pre-processing for that image. We use NumPy method from Skimage package in python coding to enhance the quality of image with respect to brightness and contrast of the existing image. This method will make pixels brighter than earlier. The result of pre-processed image compared to original image is shown in figure 12.

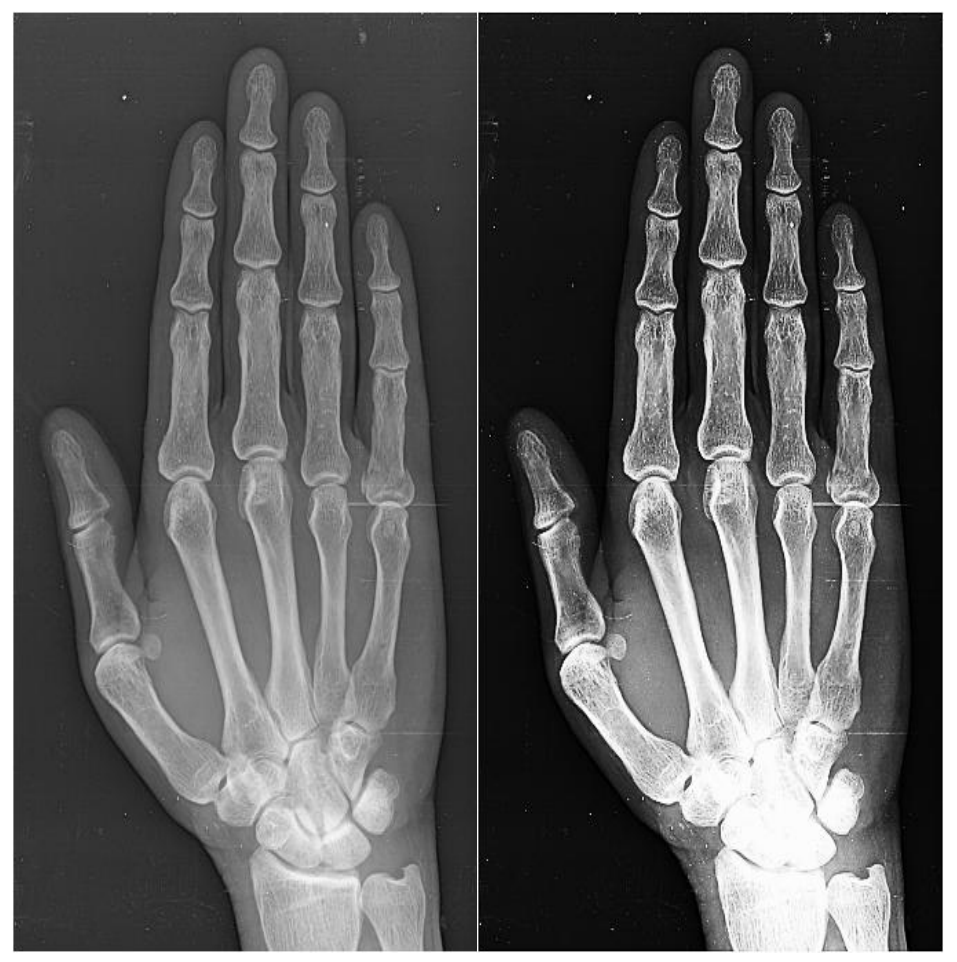

$\begin{array}{ll}\text { (a) Original image } & \text { (b) Pre-processed image }\end{array}$

Figure 12: Image pre-processing.

\section{Detection of edge using canny algorithm}

Canny edge detection algorithm is used for image segmentation. Image segmentation is the process of extracting the required image by removing the background outliers in the original image. We have used the following algorithm for edge detection.

\section{Steps for detection of edge using canny algorithm:}

1. Convolution of an image by using Gaussian Filter coefficient, Threshold value.

[T_Low $=0.075 ;]$

$\left[T_{-}\right.$High $\left.=0.175 ;\right]$

2. Convolution of image from horizontal and vertical filter

Filtered_X $=\operatorname{conv2}\left(A, K G x,{ }^{\prime}{ }^{\prime}\right)$;

Filtered_Y $=\operatorname{conv2}\left(A, K G y,{ }^{\prime}{ }^{\prime}\right)$; 
3. Calculate directions/orientations

arah $=$ atan2 $($ Filtered_Y,Filtered_X);

$\operatorname{arah}=\operatorname{arah}^{*} 180 / p i ;$

4. Calculate magnitude

magnitude $=($ Filtered_X.^2) $+($ Filtered_Y.^2 $)$;

magnitude2 = sqrt(magnitude);

5. Hysteresis Thresholding

$T \_L o w=T \_L o w * \max (\max (B W))$;

T_High = T_High $* \max (\max (B W))$;

$T \_r e s=z e r o s(p a n, l e b)$;

Result of input digital image of hand wrist, after applying canny edge algorithm process is depicted in figure 13. By this segmentation process, few important features and characters were marked and analysed that will help model to predict accurately.

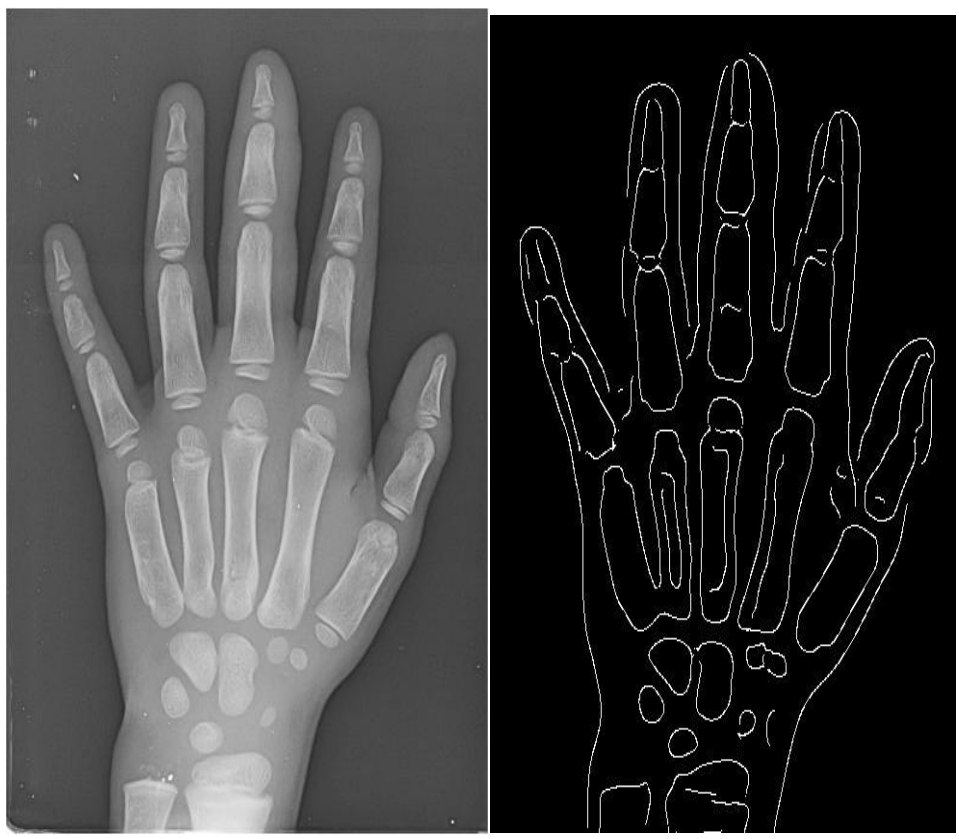

Figure 13: Detection of edge of a hand wrist bone using canny edge detector.

The hand wrist digital input image used for prediction of age and gender has to be trained. We have used Keras libraries in python platform to train and classify our data. Datasets which are collected from a hospital is stored in our local system as trained and testing folders. The training process is done through 'Train classifier' button which is provided in GUI. Once the image is trained successfully, it can be uploaded for further processes in age and gender determination. Figure 14(a) depicts the chart of male datasets with base age and figure 14(b) depicts the chart of age and gender after prediction. 


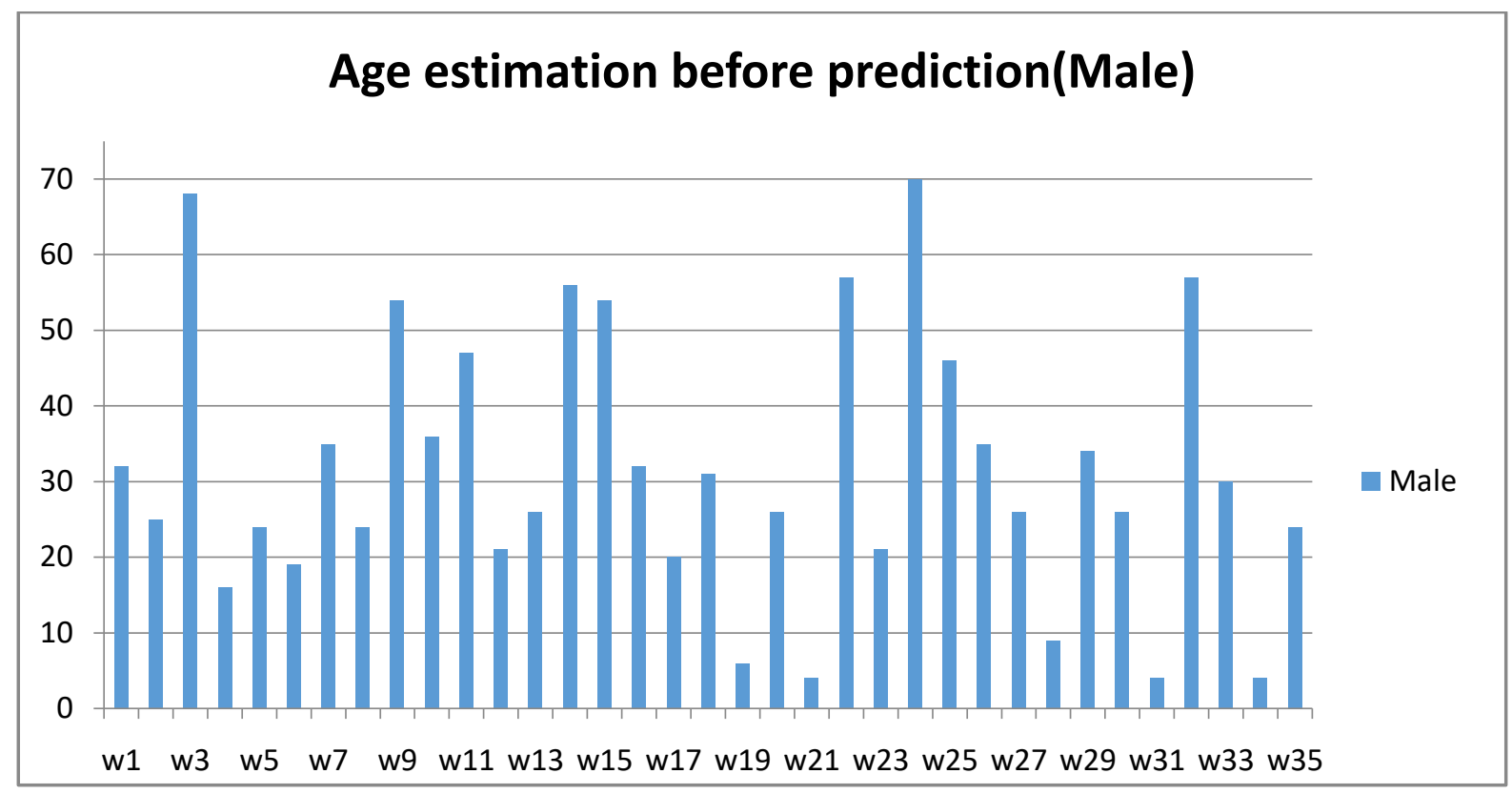

Figure 14 (a): Age and gender before prediction.

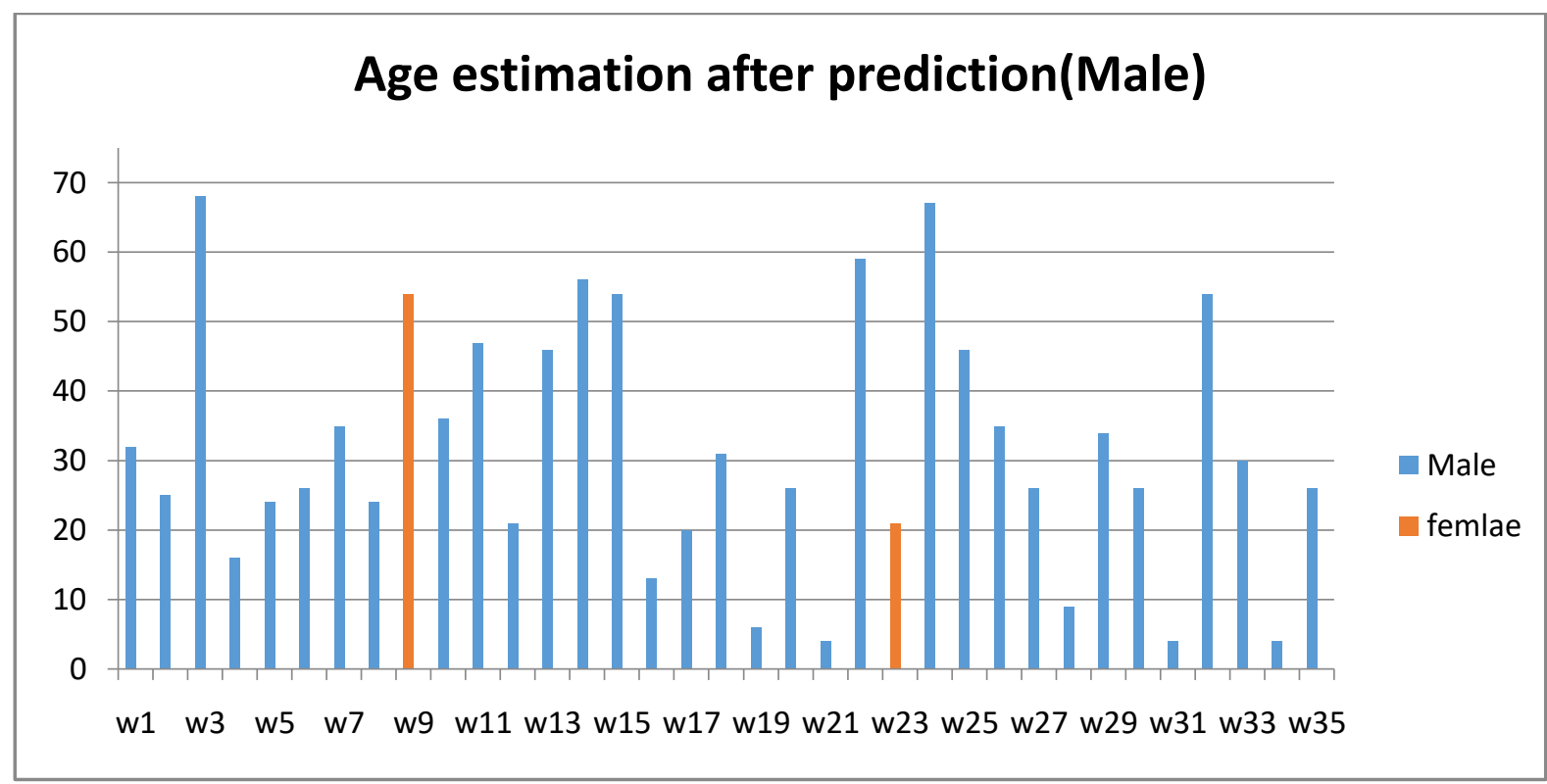

Figure 14(b): Age and gender predicted by system.

Gender determination for sample label 'w9' and sample label ' $w 24$ ' has predicted false result as female, since all the sampales are male, and age prediction in sample labelled 'w25', 'w13', 'w34' and 'w11' were miss predicted. 


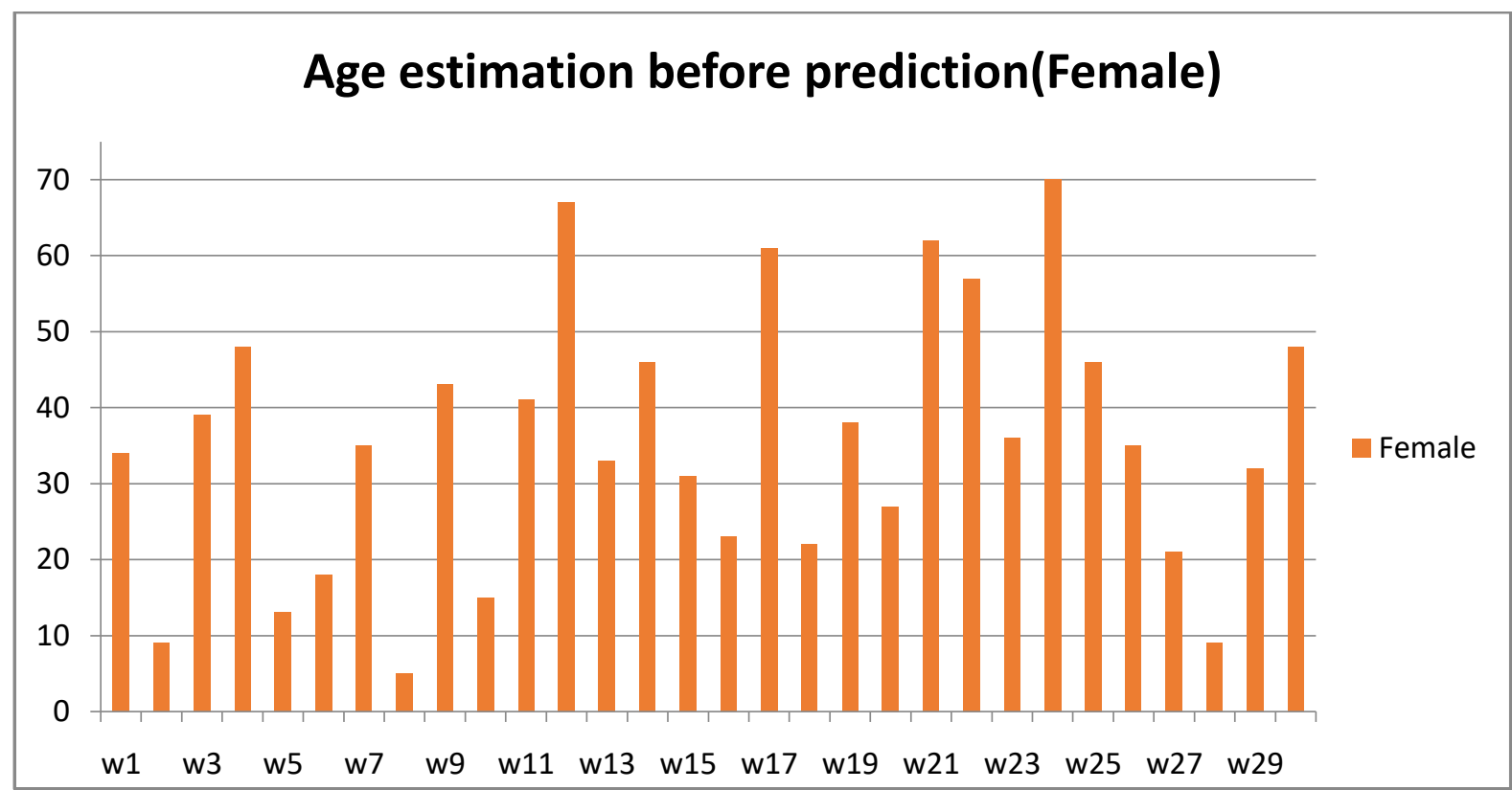

Figure 15 (a): Age and gender before prediction(Female).

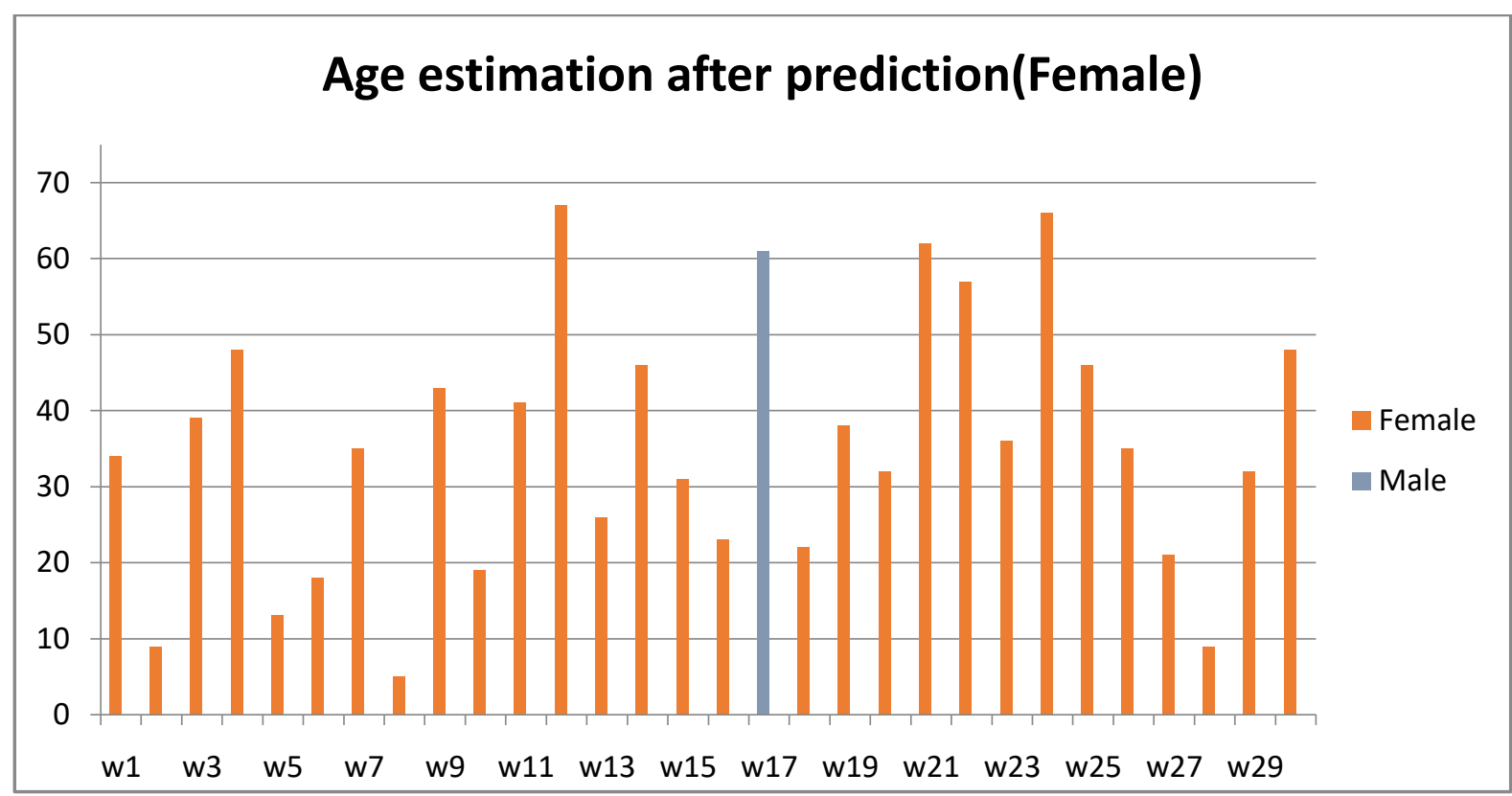

Figure 15 (b): Age and gender after prediction (Male).

Similarly figure 15(a) depicts the chart for female datasets with base age and figure 15(b) depicts the chart of age and gender after prediction. Estimated accuracy of our research experiment is $97.1 \%$ for both gender prediction and age estimation. Gender determination for sample label 'w17' has predicted false result as male, since all the samples are female, and age prediction in sample labelled 'w10', 'w13', 'w21' and 'w24' were miss predicted. Where sample label ' $\mathrm{w} 1$ ' or ' $\mathrm{w} 2$ ' is the name assigned to the dataset image in the database folder as 'wrist no. 01' 'wrist no. 02' so on.

\section{Conclusion And Future Scope}

Considering digital images of hand wrist bone, gender and age assessment is examined and studied different techniques in bone age assessment method. Age and gender dominant features are identified and extracted from hand wrist $x$ ray images. Overall outcome of the results were evaluated on the basis of true positive, true negative, false negative and false positive to judge accuracy level. Our automated method for gender and age estimation can be used to deal legal issues in identification purpose for a specific population. Our experimental results demonstrated very less variance in gender and age prediction. 
Gender and Age prediction model presented in our study is the more accurate noticed till date in Middle-East population of Karnataka, India. The prediction model can be further used as a novel system for human identification with consent of Human Rights Committee. Our present study finds its application in forensic science department where identification of human process was done manually. Further this work can be implemented as mobile applications or as web based applications which can be run on any platform. Age model accuracy can be improved with few modifications by training datasets in existing model. This work can be expanded by standardizing the dataset, identifying few more unique features that were not able to find as of now.

\section{References}

Gaskin CM, Kahn SL, Bertozzi JC, Bunch PM. "Skeletal development of the hand and wrist: a radiographic atlas and digital bone age companion”. Oxford: Oxford University Press; 2011.

Hyunkwang Lee, Shahein Tajmir, Jenny Lee, Maurice Zissen, Bethel Ayele Yeshiwas, Tarik K. Alkasab, Garry Choy, Synho Do. "Fully Automated Deep Learning Systemfor Bone Age Assessment”. J Digit Imaging 30:427-441. 2017.

M.S. Reynoldsa, *, D.M. MacGregora, C.L. Alston-Knoxb, L.S. Gregory. "Quantifying the ossification of the carpus in skeletal age estimation: Radiographic standards for Australian subadults" Forensic Science International. 2019

Mohd Asyraf Zulkifley, Siti Raihanah Abdani and Nuraisyah Hani Zulkifley. “Automated Bone Age Assessment with Image Registration Using Hand X-ray Images” Appl. Sci. 2020.

Christian Booz, Ibrahim Yel, Julian L. Wichmann, Sabine Boettger, Ahmed Al Kamali. “Artificial intelligence in bone age assessment: accuracy and efficiency of a novel fully automated algorithm compared to the GreulichPyle method", European Radiology Experimental 4:6- 2020.

Roberto Scendon, Mariano C, Andrea G, Marco F, Piergiorgio F. “Analysis of carpal bones on MR images for age estimation: first results of a new forensic approach”. Forensic Science International. 2020.

Jang Hyung Lee, Young Jae Kim, Kwang Gi Kim. "Bone age estimation using deep learning and hand X-ray images" Biomedical Engineering Letters. 10:323-331. 2020.

Ana Luiza Dallora, Peter AnderbergID, Ola K, Emilia M, Sandra D R, Johan S. "Bone age assessment with various machine learning techniques: A systematic literature review and meta-analysis". 2019.

Sehyo Yune, H Lee, Myeongchan K, Michael G. "What Machines Can Read: Gender Identification from Hand and Wrist Radiographs in Children". 2019.

Akanksha Sharma and Prabhjeet Kaur. "Hand Wrist X-Ray Images in Bone Age Assessment Using Particle Swarm and Convolutional Neural Network Algorithm" International Journal of Innovative Technology and Exploring Engineering (IJITEE). Volume-8 Issue-8, June, 2019 